\title{
Greater number of group identifications is associated with lower odds of being depressed: evidence from a Scottish community sample
}

\author{
Fabio Sani $^{1} \cdot$ Vishnu Madhok $^{3} \cdot$ Michael Norbury ${ }^{2,4} \cdot$ Pat Dugard $^{1} \cdot$ \\ Juliet R. H. Wakefield ${ }^{1}$
}

Received: 10 November 2014/ Accepted: 25 May 2015/Published online: 10 June 2015

(c) Springer-Verlag Berlin Heidelberg 2015

\begin{abstract}
Purpose Group identification has been shown to be associated with reduced risk of depression, but this research has important limitations. Our aim was to establish a robust link between group identification and depression whilst overcoming previous studies' shortcomings.

Methods 1824 participants, recruited from General Practice throughout Scotland, completed a questionnaire measuring their identification with three groups (family, community, and a group of their choice), as well as their intensity of contact with each group. They also completed a self-rated depression measure and provided demographic information. Their medical records were also accessed to determine if they had been prescribed antidepressants in the previous 6 months.

Results The number of group identifications was associated with both lower self-rated depression and lower odds of having received a prescription for antidepressants, even after controlling for the number of contact-intensive groups, level of education, gender, age, and relationship status.

Conclusions Identifying with multiple groups may help to protect individuals against depression. This highlights
\end{abstract}

Electronic supplementary material The online version of this article (doi:10.1007/s00127-015-1076-4) contains supplementary material, which is available to authorized users.

\section{Fabio Sani}

f.sani@dundee.ac.uk

1 School of Psychology, University of Dundee, Dundee DD1 4HN, Scotland, UK

2 NHS Lothian, Edinburgh, Scotland, UK

3 Park House Surgery, Bagshot, Surrey, UK

4 Present Address: Raven Song Community Health Centre, Vancouver, Canada the potential importance of social prescriptions, where health professionals encourage a depressed patient to become a member of one or more groups with which the patient believes he/she would be likely to identify.

Keywords Group identification - Social determinants of health $\cdot$ Health $\cdot$ Depression $\cdot$ Antidepressants

\section{Introduction}

Participation in the life of one or more social groups is a core dimension of human experience [1,2]. People grow up in families, tribes, villages, and communities, work in groups and organisations, play in teams and clubs, and socialise with groups of friends and acquaintances. However, group membership is not merely about being engaged in group-related activities and interacting with in-group members. Group membership involves a subjective dimension too. Specifically, it may involve feelings of belonging, affiliation, and connectedness to the group, together with a sense of sharing aspects such as values, rituals, and sentiments with other in-group members. Researchers have conceptualised this web of cognitions and feelings as group identification [3].

Group identification may have important mental health benefits. More specifically, social, developmental, and clinical psychologists have found that identification with groups such as the family [4], the professional group [5], the support group [6], and the school [7] predicts lower levels of depressive symptomatology. Indeed, in a systematic review of 13 studies assessing the relationship between identification with a given group and self-reported depression, Cruwys and colleagues [8] found the typical Pearson's correlation coefficient value to approximate -0.30 . 
Nonetheless, existing research investigating the relationship between group identification and depression has some important limitations. First, studies have generally focussed on identification with only one group. When multiple groups have been taken into account, researchers have operationalised group identification in terms of whether one declares oneself to be a member of a given group $[9,10]$. While this can be used as a proxy measure, it constitutes a relatively crude indicator of group identification [10]. Second, all studies have assessed depression through self-report measures, ignoring assessments and diagnoses made by psychiatrists and clinicians. Third, most studies have failed to control for important predictors of depression, such as socioeconomic status and relationship status. Finally, with the only exception being a study conducted by Cruwys and colleagues [10], studies have involved relatively small convenience samples.

Our aim in the present study is to establish a robust link between group identification and depression whilst overcoming the limitations of previous studies. To address the first limitation, we considered three social groups (family, local community, and a group chosen by the participant) and assessed identification with each group using a validated, multi-item instrument. This means that our predictor was the number of group identifications (rather than either one's identification with a single group, or the number of groups of which one declares oneself to be a member). To address the second limitation, we not only used a self-report measure of depression, but also checked participants' medical records to ascertain whether they had been prescribed antidepressants recently. To address the third limitation, we controlled for a number of important demographic variables such as gender, age, level of education, and relationship status. Importantly, we also controlled for the number of groups (out of the three under consideration) with which the participant had intensive contact. This is because in two studies-one involving the family and another involving the professional groupSani and colleagues [5] found that the association between group identification and depression was stronger than (and largely independent of) the association between the intensity of contact with in-group members and depression. We believed it was important to confirm these findings. To address the fourth limitation, we collected our data from a large and heterogeneous community sample.

\section{Method}

\section{Participants and procedure}

This study is based on the Scottish portion of the data obtained for Wave 1 of the two-wave cross-national Health in Groups project. Five general practitioner (GP) surgeries located in both urban and rural parts of Scotland posted participation invitations to all their patients over 18 years of age, except those with learning difficulties, terminal illnesses, or conditions such as Alzheimer's disease, dementia, or schizophrenia $(N=21,165)$. Interest in participating was expressed by 2508 patients, who returned the reply slip included with the invitation. These patients were sent a Wave 1 questionnaire, which was completed and returned by 1824 patients (770 males, 1054 females, $M_{\text {age }}=57.55$ years, $\mathrm{SD}=14.57$, range $18-97$ years), who are referred to as participants from now on. Once the questionnaires were returned, the fifth author visited the five GP surgeries and extracted the relevant health data for each participant from the GPs' computerised databases. This paper only deals with some of the variables that were included in the questionnaire and some of the data that were collected from the medical databases: for further analyses, see Sani et al. [3] and Wakefield et al. [11].

\section{Questionnaire measures}

\section{Group identifications}

To assess group identification, we used the Group Identification Scale (GIS) [3]. This is a global scale based on four items tapping one's sense of belonging to the group (e.g. "I have a sense of belonging to [my group]") and one's sense of commonality with in-group members (e.g. "I have a lot in common with the members of [my group]"). Participants specify their disagreement or agreement with each item using a seven-point scale $(1=$ "strongly disagree", 7 = "strongly agree"). After calculating the average score on the four items, a respondent is classified as being either not identified with the group, if their score is less than 5, or as identified with the group, if their score is 5 or above. See [3] for the rationale for GIS, as a well as three independent studies assessing its internal reliability, convergent and divergent validity, and temporal stability.

The GIS instrument was used with reference to three social groups, namely the family, the local community, and an in-group chosen by the participant. Participants were instructed to define 'family' "in any way you wish (e.g. immediate family or extended family)", and 'local community' as "your neighbourhood, village, city area, or any other way you may define it". The chosen group was selected from a list which included social groups such as sports team, group of friends, hobby group, religious group, or voluntary group.

The number of group identifications for each participant was then counted. This number ranged from 0 (indicating that the participant did not identify with any of the three groups) to 3 (indicating that the participant identified with all three groups). 


\section{Contact-intensive groups}

For each of the three social groups considered (family, local community, and chosen group), we asked three questions assessing the extent to which participants interacted with other in-group members and participated in group-related activities. The first two questions were identical for all three social groups: "On average, with how many different members of your [group] do you have a face-to-face conversation in a single week?" and "On average, with how many different members of your [group] do you have a telephone/Internet conversation in a single week?" The third question differed depending on group type. Concerning the family, we asked: "On average, how many family-related events (for instance meals out, parties, gatherings, trips, etc.) do you attend in a single month?" Concerning the local community, we asked: "On average, how many local community-related events (for instance, parties, gatherings, trips, fund-raising events, etc.) do you attend in a single year?" Finally, concerning the chosen group, we asked: On average, how many events related to your chosen group (for instance, parties, gatherings, trips, etc.) do you attend in a single year?"

Then, for each of the three social groups, we transformed each participant's responses to the three contact questions into $Z$-scores, and then summed these $Z$-scores into an overall measure of contact. Concerning this overall measure, a group was considered to be either not contact intensive for the participant, when the participant scored below 0 (less than average contact), or contact intensive for the participant, when the participant scored 0 or more (average/more than average contact). Finally, for each participant we counted the number of contact-intensive groups. This variable ranged from 0 (indicating that the participant did not have any contact-intensive groups) to 3 (indicating that the participant had intensive contact with all three groups).

For details of how we handled missing data with reference to the above measures (i.e. group identifications and contact-intensive groups), see Appendix 1 in the supplementary material of Sani et al. [3].

\section{Depression}

The Major Depression Inventory (MDI) [12, 13] was used to assess the presence of depressive symptomatology. The MDI includes 12 items tapping various depressive symptoms (e.g. 'Felt low in spirits or sad'). Participants rate how often they have experienced each symptom in the past 2 weeks, using a six-point scale ranging from 0 ('at no time') to 5 ('all of the time'). Severity of depressive symptoms is calculated by summing scores on individual items. However, only the highest score is used for two pairs of items (one pair refers to feeling either restless or slowed down, and the other pair refers to suffering from either reduced or increased appetite). This means that only ten items are used to calculate the total score, which therefore can range from 0 to 50. Participants scoring 20 or more are classified as suffering from depression [14].

Participants who failed to respond more than two items out of ten were not included in the analysis. When a participant had either one or two missing responses, we replaced each missing response with the mean value of the participant's valid responses.

\section{Demographic variables}

As well as recording gender and age, we also asked participants to indicate the highest level of education they had obtained. We created a binary variable distinguishing those with up to high school education from those with any qualification above high school. We also assessed whether or not the participant was in a relationship (marriage, civil partnership, or informal partnership) at the time of questionnaire completion.

\section{Medical data}

\section{Antidepressant prescriptions}

A search was performed on the GPs' medical databases to determine the number of prescriptions for antidepressant drugs each participant had received from a health-care professional in the 6 months prior to the day of medical data collection. 'Antidepressant drugs' were defined as any drug appearing in the chapter "Antidepressant drugs" of the British National Formulary [15] and includes "tricyclic and related antidepressant drugs, monoamine-oxidase inhibitors, selective serotonin reuptake inhibitors, and other antidepressant drugs." A binary variable (antidepressants) was created to differentiate between participants who had been prescribed no antidepressants in the last 6 months and those who had been prescribed at least one antidepressant in the last 6 months.

\section{Statistical analyses}

Analyses were conducted using Statistical Package for the Social Sciences (SPSS) version 21. First, we conducted cross-tabular analyses exploring the across-gender frequencies of group identifications, contact-intensive groups, education, relationship status, depression, and antidepressant prescriptions. Subsequently, further cross-tabular analyses were conducted to investigate the frequency of participants with depression and the frequency of participants who had been prescribed antidepressants, both as a 
function of the number of group identifications and the number of contact-intensive groups. We calculated the statistical significance of differences in these frequencies using Pearson Chi square. If statistically significant effects emerged for a specific analysis, we conducted post hoc comparisons to further explore the nature of the differences. Finally, we performed two direct binary logistic regressions assessing: (1) the effects of the number of group identifications on both depression and antidepressants and (2) the effects of the number of contact-intensive groups on both depression and antidepressants. In both analyses, we controlled for gender, age, education, and relationship status. These logistic regressions produced, among other useful values, odds ratios, which are excellent indicators of effect size. An odds ratio (OR) refers to the odds that an outcome (e.g. depression) will occur given a particular exposure (e.g. isolation), compared to the odds of the outcome occurring in the absence of that exposure (e.g. multiple group identifications) [16].

\section{Results}

\section{Cross-tabular analyses}

We found that $44.78 \%$ of participants identified with three groups, while only $5.17 \%$ had no identifications. Women tended to have more group identifications than men, $\chi^{2}$ (3, $N=1800)=31.16, p<0.001$. Only $10.21 \%$ of participants had three contact-intensive groups, while most participants had either none $(37.68 \%)$ or one $(31.98 \%)$. No gender differences emerged on this variable, $\chi^{2}$ (3, $N=1704)=3.27, p=0.35$. The majority of participants were educated to above high school level $(63.61 \%)$ and were in a relationship $(75.23 \%)$. However, women were more likely to be educated to above high school level than men, $\chi^{2}(1, N=1811)=8.52, p=0.004$, while men were more likely to be in a relationship than women, $\chi^{2}$ (1, $N=1813)=12.20, p<0.001$. Finally, we found that $8.26 \%$ of participants were depressed, with no gender differences emerging, $\chi^{2}(1, N=1816)=0.01, p=0.91$, and that $9.53 \%$ of participants had been prescribed antidepressants in the last 6 months, with a statistically significant difference between men $(6.64 \%$ of whom had received a prescription) and women (11.65\% of whom had received a prescription), $\chi^{2}(1, \quad N=1815)=12.90$, $p<0.001$. See Appendix A in the supplementary material for details of these analyses.

Concerning the relationship between the number of group identifications and the two depression indicators, analyses showed that as the number of group identifications increased, the proportion of both participants with depression and participants who had been prescribed antidepressants decreased, with the relationship following a clear gradient. Specifically, $44.57 \%$ of respondents without any group identifications were depressed, compared to $17.14,5.67$ and $2.36 \%$ of respondents with one, two, and three group identifications, respectively. Furthermore, $20.43 \%$ of respondents without any group identifications had received a prescription for antidepressants in the last 6 months, compared to $12.74,9.47$, and $6.85 \%$ of respondents with one, two, and three group identifications, respectively. The association between the number of group identifications and self-rated depression, $\chi^{2} \quad(3$, $N=1795)=236.87, \quad p<0.001$, and the association between the number of group identifications and antidepressants, $\chi^{2}(3, N=1791)=23.45, p<0.001$, were both statistically significant. See Table 1 for details.

At this point, we conducted post hoc comparisons for each outcome (depression and antidepressants): see Table 2 for results [17]. We found that the depression frequency for participants with at least one group identification was lower than for those with no group identifications. It was also lower for those with two or three group identifications than for those with only one group identification. Finally, it was lower for those with three group identifications than for those with two group identifications ( $p s<0.001)$. We also found that the antidepressant frequency for participants with at least one group identification was lower than for those with no group identifications $(p<0.001)$. It was also lower for those with two or three group identifications than for those with only one group identification $(p=0.007)$. Finally, it was also lower for those with three group identifications than for those with two group identifications, although this result was only marginal $(p=0.076)$.

Regarding the relationship between contact-intensive groups and the two depression indicators, analyses revealed that as the number of contact-intensive groups increased, the proportion of participants with self-rated depression decreased, with the relationship following a clear gradient. Specifically, $12.54 \%$ of respondents without any contactintensive groups had depression, compared to $6.99,3.21$, and $2.30 \%$ of respondents with one, two, and three contact-intensive groups, respectively. This association was statistically significant, $\chi^{2} \quad(3, \quad N=1699)=37.69$, $p<0.001$. However, concerning the relationship between the number of contact-intensive groups and antidepressants, the pattern was less clear, with 11.56, 8.91, 7.31, and $8.05 \%$ of participants with zero, one, two, and three contact-intensive groups having been prescribed antidepressants, respectively. This association did not reach statistical significance, $\chi^{2}(3, N=1695)=5.73, p=0.13$. See Table 3 for details of these analyses.

We conducted post hoc comparisons for depression (the significant Chi-square result). We found the depression 
Table 1 Frequencies and percentages for depressed and antidepressants at each level of group identifications $(0-3)$, including Chi square values

\begin{tabular}{llllll}
\hline Group identifications & \multicolumn{2}{l}{ Depressed (self-rated) } & & \multicolumn{2}{l}{ Antidepressants } \\
\cline { 2 - 3 } \cline { 5 - 5 } \cline { 5 - 5 } & No & Yes & & No & Yes \\
\hline 0 & 51 & 41 & 74 & 19 \\
$(n=93)$ & $(55.43 \%)$ & $(44.57 \%)$ & & $(79.57 \%)$ & $(20.43 \%)$ \\
1 & 261 & 54 & 274 & 40 \\
$(n=316)$ & $(82.86 \%)$ & $(17.14 \%)$ & & $(87.26 \%)$ & $(12.74 \%)$ \\
2 & 549 & 33 & 526 & 55 \\
$(n=585)$ & $(94.33 \%)$ & $(5.67 \%)$ & & $(90.53 \%)$ & $(9.47 \%)$ \\
3 & 787 & 19 & 748 & 55 \\
$(n=806)$ & $(97.64 \%)$ & $(2.36 \%)$ & & $(93.15 \%)$ & $(6.85 \%)$ \\
& $\chi^{2}(3, N=1795)=236.87 ; p<0.001$ & & $\chi^{2}(3, N=1791)=23.45 ; p<0.001$ \\
& Cramer's $V=0.36, p<0.001$ & & Cramer's $V=0.11, p<0.001$ \\
\hline
\end{tabular}

24 participants had a missing value for group identifications. These cases are excluded from the relevant sections of this table. Missing values prevent frequencies in the table always summing to match the overall $N \mathrm{~s}$ in the first column

Table 2 Post hoc comparisons following results of Chi square analyses of depressed and antidepressant frequencies as a function of number of group identifications

\begin{tabular}{|c|c|c|c|c|}
\hline \multirow[t]{2}{*}{ No. of group identifications } & \multicolumn{2}{|c|}{ Depressed (self-rated) } & \multicolumn{2}{|c|}{ Antidepressants } \\
\hline & No & Yes & No & Yes \\
\hline 0 & $\begin{array}{l}51 \\
(55.43 \%)\end{array}$ & $\begin{array}{l}41 \\
(44.57 \%)\end{array}$ & $\begin{array}{l}74 \\
(79.57 \%)\end{array}$ & $\begin{array}{l}19 \\
(20.43 \%)\end{array}$ \\
\hline 1,2 , or 3 & \multicolumn{2}{|c|}{$\begin{array}{l}\chi^{2}(1, N=1795)=170.65 ; p<0.001 \\
\varphi=-0.31, p<0.001\end{array}$} & \multicolumn{2}{|c|}{$\begin{array}{l}\chi^{2}(1, N=1791)=13.87 ; p<0.001 \\
\varphi=-0.09, p<0.001\end{array}$} \\
\hline 1 & $\begin{array}{l}261 \\
(82.86 \%)\end{array}$ & $\begin{array}{l}54 \\
(17.14 \%)\end{array}$ & $\begin{array}{l}274 \\
(87.26 \%)\end{array}$ & $\begin{array}{l}40 \\
(12.74 \%)\end{array}$ \\
\hline \multirow[t]{2}{*}{2 or 3} & $\begin{array}{l}1336 \\
(96.25 \%)\end{array}$ & $\begin{array}{l}52 \\
(3.75 \%)\end{array}$ & $\begin{array}{l}1274 \\
(92.05 \%)\end{array}$ & $\begin{array}{l}110 \\
(7.95 \%)\end{array}$ \\
\hline & \multicolumn{2}{|c|}{$\begin{array}{l}\chi^{2}(1, N=1703)=78.94 ; p<0.001 \\
\varphi=-0.22, p<0.001\end{array}$} & \multicolumn{2}{|c|}{$\begin{array}{l}\chi^{2}(1, N=1698)=7.29 ; p=0.007 \\
\varphi=-0.07, p=0.007\end{array}$} \\
\hline 2 & $\begin{array}{l}549 \\
(94.33 \%)\end{array}$ & $\begin{array}{l}33 \\
(5.67 \%)\end{array}$ & $\begin{array}{l}526 \\
(90.53 \%)\end{array}$ & $\begin{array}{l}55 \\
(9.47 \%)\end{array}$ \\
\hline \multirow[t]{2}{*}{3} & $\begin{array}{l}787 \\
(97.64 \%)\end{array}$ & $\begin{array}{l}19 \\
(2.35 \%)\end{array}$ & $\begin{array}{l}748 \\
(93.15 \%)\end{array}$ & $\begin{array}{l}55 \\
(6.85 \%)\end{array}$ \\
\hline & \multicolumn{2}{|c|}{$\begin{array}{l}\chi^{2}(1, N=1388)=10.29 ; p=0.001 \\
\varphi=-0.09, p=0.001\end{array}$} & \multicolumn{2}{|c|}{$\begin{array}{l}\chi^{2}(1, N=1384)=3.16 ; p=0.076 \\
\varphi=-0.05, p=0.076\end{array}$} \\
\hline
\end{tabular}

frequency for participants with at least one contact-intensive group was lower than for participants with no contactintensive groups $(p<0.001)$. It was also lower for those with two or three contact-intensive groups than for those with only one contact-intensive group $(p=0.002)$. However, it was not lower for those with three contact-intensive groups than for those with two contact-intensive groups $(p=0.56)$.

For a table of mean scores on the Major Depression Inventory as a function of number of group identifications and number of contact-intensive groups, see Appendix B in the supplementary material.

\section{Logistic regression analyses}

\section{Assumptions}

Before running the logistic regression analyses, we checked whether the data met the necessary assumptions. First, we assessed the linearity of the logit for our 
Table 3 Frequencies and percentages for depressed and antidepressants at each level of contact-intensive groups (0-3), including Chi-square values

\begin{tabular}{llllll}
\hline Contact-intensive groups & \multicolumn{2}{l}{ Depressed (self-rated) } & & \multicolumn{2}{l}{ Antidepressants } \\
\cline { 2 - 3 } \cline { 5 - 5 } \cline { 5 - 5 } & No & Yes & & No & Yes \\
\hline 0 & 558 & 80 & 566 & 74 \\
$(N=642)$ & $(87.46 \%)$ & $(12.54 \%)$ & & $(88.44 \%)$ & $(11.56 \%)$ \\
1 & 506 & 38 & & 491 & 48 \\
$(N=545)$ & $(93.01 \%)$ & $(6.99 \%)$ & & $(91.09 \%)$ & $(8.91 \%)$ \\
2 & 332 & 11 & & 317 & 25 \\
$(N=343)$ & $(96.79 \%)$ & $(3.21 \%)$ & & $(92.69 \%)$ & $(7.31 \%)$ \\
3 & 170 & 4 & & 160 & 14 \\
$(N=174)$ & $(97.70 \%)$ & $(2.30 \%)$ & & $(91.95 \%)$ & $(8.05 \%)$ \\
& $\chi^{2}(3, N=1699)=37.69 ; p<0.001$ & & $\chi^{2}(3, N=1695)=5.73 ; p=0.13$ \\
& Cramer's $V=0.15, p<0.001$ & & Cramer's $V=0.06, p=0.13$ \\
\hline
\end{tabular}

120 participants had a missing value for contact-intensive groups. These cases are excluded from the relevant sections of this table. Missing values prevent frequencies in the table always summing to match the overall $N \mathrm{~s}$ in the first column continuous predictors (i.e. group identifications, contactintensive groups, and age). This involved running both logistic regressions with three additional interaction terms in each analysis (i.e. the interaction between each continuous variable and its own log). Only one of these interaction terms was statistically significant in one analysis: that for age when the antidepressants variable was being predicted. Removing this covariate from the analysis did not alter the pattern of our results.

We then tested the data for multicollinearity. Tolerance values ranged from 0.78 to 0.96 , while the highest variance inflation factor value was 1.27 , clearly indicating a lack of multicollinearity. Finally, we investigated outliers. In neither of the two logistic regressions did the number of cases with Studentised residual values greater than two cause concern. On the basis of these results, we proceeded with the two logistic regression analyses.

\section{Analyses}

The first logistic regression focussed on the impact of the predictors (number of group identifications, number of contact-intensive groups, gender, age, education, and relationship status) on the odds that participants self-rated as depressed. The number of group identifications was a strong predictor of depression, with every additional group identification markedly decreasing the odds of being depressed, OR 0.32, $p<0.001$. Furthermore, having education above high school (vs. high school or less), being in a relationship (vs. not being in a relationship), and being older (vs. being younger) predicted lower odds of depression, OR 0.50, $p=0.001$; OR $0.49, p=0.001$; and OR $0.97, p<0.001$, respectively. The remaining two predictors - the number of contact-intensive groups and gender- did not have a statistically significant impact on depression. See Table 4 for full results.

The second logistic regression looked at the impact of the predictors (number of group identifications, number of contact-intensive groups, gender, age, education, and relationship status) on the odds that participants had been prescribed antidepressants in the previous 6 months. A greater number of group identifications predicted lower odds of having been prescribed antidepressants, OR 0.70 , $p<0.001$. The odds of being prescribed antidepressants were also markedly decreased by being in a relationship (vs. not being in a relationship), OR $0.57, p=0.002$, and by being male (vs. being female), OR $0.52, p<0.001$. None of the other predictors had a statistically significant impact on the odds of being prescribed antidepressants. See Table 5 for full results.

For a hierarchical logistic regression analysis assessing the variance in the outcomes explained by group identifications and contact-intensive groups in addition to the variance explained by the control variables, see Appendix $\mathrm{C}$ in the supplementary material.

\section{Discussion}

The study presented in this paper demonstrates that greater number of group identifications is associated with both lower odds of self-rated depression and lower odds of having received a prescription for antidepressants in the last 6 months, even after controlling for the number of contact-intensive groups, level of education, gender, age, and whether or not one is in a relationship.

These findings are consistent with Cruwys et al.'s [8] assertion that group identification is antithetical to 
Table 4 Summary of logistic regression analysis for variables predicting depressed (self-rated)

\begin{tabular}{lllclccc}
\hline Variable & $B$ & SE & Wald statistic & $p$ & Odds ratio & \multicolumn{2}{c}{ 95 \% CI for odds ratio } \\
\cline { 5 - 8 } & & & & & & Lower & Upper \\
\hline Group identifications (0-3) & -1.16 & 0.12 & $87.52^{* * *}$ & $<0.001$ & 0.32 & 0.25 & 0.40 \\
Contact-intensive groups (0-3) & -0.07 & 0.13 & 0.27 & 0.61 & 0.93 & 0.72 & 1.21 \\
Education (0/1) & -0.69 & 0.21 & $11.48^{* *}$ & 0.001 & 0.50 & 0.33 & 0.75 \\
Relationship status (0/1) & -0.72 & 0.21 & $11.90^{* *}$ & 0.001 & 0.49 & 0.33 & 0.73 \\
Gender (0/1) & -0.19 & 0.21 & 0.86 & 0.35 & 0.83 & 0.55 & 1.24 \\
Age (years) & -0.03 & 0.01 & $21.06^{* * *}$ & $<0.001$ & 0.97 & 0.96 & 0.98 \\
\hline
\end{tabular}

$* * * p<0.001$

$* * p<0.01$

Table 5 Summary of logistic regression analysis for variables predicting antidepressants

\begin{tabular}{lllccccc}
\hline Variable & $B$ & SE & Wald statistic & $p$ & Odds ratio & \multicolumn{2}{c}{$95 \%$ CI for odds ratio } \\
\cline { 3 - 7 } & & & & & & Lower & Upper \\
\hline Group identifications (0-3) & -0.36 & 0.10 & $12.65^{* * *}$ & $<0.001$ & 0.70 & 0.57 & 0.85 \\
Contact-intensive groups (0-3) & -0.03 & 0.10 & 0.08 & 0.78 & 0.97 & 0.80 & 1.18 \\
Education (0/1) & -0.05 & 0.18 & 0.09 & 0.77 & 0.95 & 0.67 & 1.35 \\
Relationship status (0/1) & -0.57 & 0.18 & $9.96^{* *}$ & 0.002 & 0.57 & 0.40 \\
Gender (0/1) & -0.66 & 0.19 & $12.59^{* * *}$ & $<0.001$ & 0.52 & 0.36 & 0.81 \\
Age (years) & -0.002 & 0.01 & 0.07 & 0.79 & 1.00 & 0.99 & 1.01 \\
\hline
\end{tabular}

$* * * p<0.001$

$* * p<0.01$

depression. While group identification affords a sense of structure, purpose, and meaning, and fosters positive social relationships based on trust, support, and respect [18], depressive disorder is characterised by loss of meaning in life and inability to function socially [19]. The other grouprelated element that was considered, that is number of contact-intensive groups, was associated with self-rated depression (but not antidepressant prescriptions) in bivariate cross-tabular analysis. However, when entered into a regression analysis that also included number of group identifications, the number of contact-intensive groups did not exert any independent effect on either selfrated depression or antidepressant prescriptions. This suggests that the link between depression and intensity of social contact with in-group members observed in other studies [20] might have actually resulted from group identification stimulating and encouraging greater contact. This seems to confirm Cruwys et al.'s [10] proposal that group identification is the "active ingredient" that allows group life both to protect against depression and to favour faster and more permanent recovery from it.

It should be noted, however, that the number of group identifications was more strongly associated with self-reported depression than with antidepressant prescriptions.
Presumably, the association between group identifications and antidepressants is weakened by the fact that a substantial amount of those who feel depressed do not receive a prescription for antidepressants. Some people who are depressed may simply fail to see a doctor either because they do not recognise the problem [21] or because they opt for forms of self-medication such as consumption of alcohol [22] or nicotine [23]. Others may see a doctor, but refuse to take the antidepressants they are prescribed [24], or the doctor may decide against prescribing antidepressants because the patient's symptomatology is not considered sufficiently severe, or because of pregnancy, old age, or the presence of certain physical symptoms $[25,26]$.

\section{Limitations and future directions}

Our study is not without its limitations. Perhaps most importantly, the cross-sectional nature of our research design means that we cannot make inferences about causal processes. This means that it could be argued that depression predicts group identification, rather than group identification predicting depression. However, Cruwys et al. [8] found that the number of social groups of which participants declared themselves to be members predicted 
depression over time, but not vice versa. Although, as mentioned above, self-reported membership is not exactly equivalent to group identification, these findings are consistent with the possibility that an individual's number of group identifications determines their depression. Furthermore, Cacioppo et al. [27] found that loneliness predicted subsequent changes in depressive symptomatology, but not vice versa. These researchers conceptualised and measured loneliness in terms of one's experienced lack of companionship and isolation from others. As such, feeling lonely may be seen, in some respects at least, as the opposite of enjoying multiple group identifications. These findings are thus also consistent with the possibility that the number of group identifications influences depression. Nonetheless, we do not entirely exclude the possibility that, to some degree, an individual's depression determines their number of group identifications. We hope that the data obtained from the second wave of the Health in Groups project will help to shed light on this important issue.

A further limitation of our study concerns the fact that we only assessed participants' identification with three social groups. We recognise that individuals may potentially belong to more than three groups, but we wished to keep our questionnaire relatively short so as to maximise response rates. While it could be argued that identification with more than three social groups might be associated with even lower levels of self-rated depression and antidepressant prescriptions, we feel that the very low levels of self-rated depression (2.36 \%) and antidepressant prescriptions $(6.85 \%)$ that we obtained for participants with three group identifications in the present study suggests a floor effect, implying that additional identifications are unlikely to reduce depression and antidepressant values much further than those observed in our results. Nonetheless, it might be useful for future research to assess the relationship between mental health and participants' identification with more than three social groups.

Finally, although participation invitations were sent to all adult patients (for whom the study was deemed appropriate) who were registered at each of the five GP surgeries, the survey nature of our research design meant that participants decided for themselves whether or not to actually participate in the study. This inevitably promotes self-selection bias. It is difficult to speculate about the characteristics of those individuals that might have been more inclined to participate in the study. It is however useful to note that the proportion of participants that had been prescribed antidepressants in the 6 months preceding medical data collection was $9.53 \%$. This is approximately twothirds of the proportion of Scots (i.e. slightly less than $15 \%$ ) who were prescribed an antidepressant during 2013-2014 [28]. We only measured antidepressants prescribed up to 6 months before data collection, but it is likely that the proportion of participants prescribed antidepressants up to 1 year before data collection would be closer to $15 \%$. This suggests that our results are broadly in line with Scottish trends. Therefore, at least in terms of a crucial outcome variable such as prescriptions for antidepressants, our sample does not seem to be particularly biased.

\section{Implications and conclusions}

If confirmed by further studies, especially randomised controlled trials, these findings would constitute evidence in support of the idea of social prescriptions [29]. Specifically, a health professional could present a depressed patient with a pre-prepared list of local social groups (e.g. sports or chess club, reading group, yoga, drawing, or foreign language classes) and encourage the patient to become a member of one or more groups with which the patient believes he/she would be likely to identify. The health professional could also encourage the patient to consider contributing to the creation and development of groups of which he/she would like to be a member, and with which he/she would be likely to identify. However, we wish to emphasise that, according to our findings, merely having contact with in-group members will not produce any real beneficial effect unless that contact is framed by group identification (i.e. a sense of belonging to the group, coupled with a sense of commonality with its members). In addition, we suspect that high levels of contact with members of a group might even produce detrimental effects when people openly dislike, or actively disidentify with, the group in question.

The type of social prescription described above could be used in tandem with either medication or psychotherapy. However, a social prescription might be an especially viable option for patients who find psychotherapy unhelpful or unproductive, patients who do not want (or cannot take) antidepressants for the reasons discussed above, and patients who have tried antidepressants but have decided to discontinue taking them because of unpleasant side effects such as emotional numbing and sexual dysfunction [30, 31]. In such cases, we believe that health practitioners should consider social prescription as a key tool for mental health promotion, particularly when there are reasons to believe that a patient's depression is caused mainly by social isolation or loneliness.

In conclusion, group life is a constitutive aspect of human existence. Across prehistory and history, groups have been at the heart of activities such as finding food, defence from predation, moving across places, and defining moral and behavioural norms $[32,33]$. Work such as ours provides a tantalising glimpse of how groups might also be vital for our mental health and well-being. 
Acknowledgments This research was made possible by a research grant awarded by the Economic and Social Research Council (ESRC) to the first, second, and third authors (Grant reference number ES/ I038349/1). We were also assisted by the Scottish Primary Care Research Network (SPCRN) during data collection. The ESRC and SPCRN had no involvement in the study's design, data collection, data analysis, data interpretation, or the writing and submission of this article.

Conflict of interest On behalf of all the authors, the corresponding author states that there is no conflict of interest.

Ethical standard Our study was approved by the appropriate ethics committee and has therefore been performed in accordance with the ethical standards laid down in the 1964 Declaration of Helsinki and its later amendments.

\section{References}

1. Tomasello M (2009) The cultural origins of human cognition. Harvard University Press

2. Tuomela R (2007) The philosophy of sociality: The shared point of view. Oxford University Press

3. Sani F, Madhok V, Norbury M, Dugard P, Wakefield JRH (2014) Higher identification with social groups is associated with healthier behavior: evidence from a Scottish community sample. Br J Health Psych. doi:10.1111/bjhp.12119

4. Sani F, Magrin ME, Scrignaro M, McCollum R (2010) In-group identification mediates the effects of subjective in-group status on mental health. Br J Soc Psychol 49:883-893

5. Sani F, Herrera M, Wakefield JRH, Boroch O, Gulyas C (2012) Comparing social contact and group identification as predictors of mental health. Br J Soc Psychol 51:781-790

6. Wakefield JRH, Bickley S, Sani F (2013) The effects of identification with a support group on the mental health of people with multiple sclerosis. J Psychom Res 74:420-426

7. Bizumic B, Reynolds KJ, Turner JC, Bromhead D, Subasic E (2009) The role of the group in individual functioning: school identification and the psychological well-being of staff and students. Appl Psychol 58:171-192

8. Cruwys T, Haslam SA, Dingle GA, Haslam C, Jetten J (2014) Depression and social identity: an integrative review. Pers Soc Psychol Rev 18:215-238

9. Iyer A, Jetten J, Tsivrikos D, Postmes T, Haslam SA (2009) The more (and the more compatible) the merrier: multiple group memberships and identity compatibility as predictors of adjustment after life transitions. Br J Soc Psychol 48:707-733

10. Cruwys T, Dingle GA, Haslam C, Haslam SA, Jetten J, Morton T (2013) Social group memberships protect against future depression symptoms and prevent depression relapse. Soc Sci Med 98:179-186

11. Wakefield JRH, Sani F, Madhok V, Norbury M, Dugard P (2015) The pain of low status: the relationship between subjective socioeconomic status and analgesic prescriptions in a Scottish community sample. Psychol Health Med. doi:10.1080/13548506. 2015.1009377

12. Bech P, Rasmussen N-A, Olsen LR, Noerholm V, Abildgaard W (2001) The sensitivity and specificity of the Major Depression Inventory, using the Present State Examination as the index of diagnostic validity. J Affect Disorders 66:159-164
13. Cuijpers P, Dekker J, Noteboom A, Smits N, Peen J (2007) Sensitivity and specificity of the Major Depression Inventory in outpatients. BMC Psychiatry 7:39

14. Olsen LR, Jensen DV, Noerholm V, Martiny K, Bech P (2003) The internal and external validity of the Major Depression Inventory in measuring severity of depressive states. Psychol Med 33:351-356

15. Joint Formulary Committee (2012) British national formulary, 64th edn. BMJ Group and Pharmaceutical Press, London

16. Ellis PD (2010) The essential guide to effect sizes: Statistical power, meta-analysis, and the interpretation of research results. Cambridge University Press

17. Kendall M, Stewart A (1979) The advanced theory of statistics. In: Inference and relationship, vol 2. Hodder Arnold, London

18. Haslam SA, Jetten J, Postmes T, Haslam C (2009) Social identity, health and well-being: an emerging agenda for applied psychology. Appl Psychol 58:1-23

19. American Psychiatric Association (2014) Diagnostic and statistical manual of mental disorders, 5th edn. American Psychiatric Publishing, Arlington, VA

20. Kawachi I, Berkman LS (2001) Social ties and mental health. J Urban Health 78:458-467

21. Jorm AF (2000) Mental health literacy: public knowledge and beliefs about mental disorders. Br J Psychiatry 177:396-401

22. Bolton JM, Robinson J, Sareen J (2009) Self-medication of mood disorders with alcohol and drugs in the National Epidemiologic Survey on Alcohol and Related Conditions. J Affect Disorders 115:367-375

23. Lerman C, Caporaso N, Main D, Audrain J, Boyd NR, Bowman ED, Shields PG (1998) Depression and self-medication with nicotine: the modifying influence of the dopamine D4 receptor gene. Health Psychol 17:56-62

24. Dwight-Johnson M, Sherbourne CD, Liao D, Wells KB (2000) Treatment preferences among depressed primary care patients. J Gen Intern Med 15:527-534

25. Jones I, McDonald L (2014) Living with uncertainty: antidepressants and pregnancy. Br J Psychiatry 205:103-104

26. Kendrick T, Dowrick C, McBride A, Howe A, Clarke P, Maisey S, Moore M, Smith PW (2009) Management of depression in UK general practice in relation to scores on depression severity questionnaires: analysis of medical record data. BMJ 338:b750

27. Cacioppo JT, Hawkley LC, Thisted RA (2010) Perceived social isolation makes me sad: 5-year cross-lagged analyses of loneliness and depressive symptomatology in the Chicago Health Aging, and Social Relations Study. Psychol Aging 25:453-463

28. Scottish Government (2014) Medicines for mental health: financial years 2004/05 to 2013/14. A National Statistics Publication for Scotland, Edinburgh

29. Scottish Government (2007) Developing social prescribing and community referrals for mental health in Scotland. Scottish Development Centre for Mental Health, Edinburgh

30. Price J, Coole V, Goodwin GM (2009) Emotional side-effects of selective serotonin reuptake inhibitors: qualitative study. $\mathrm{Br} \mathrm{J}$ Psychiatry 195:211-217

31. Baldwin DS, Foong T (2013) Antidepressant drugs and sexual dysfunction. Br J Psychiatry 202:396-397

32. Caporael LR, Brewer MB (1991) Revisiting evolutionary psychology. Biology meets society. J Soc Issues 47:187-195

33. Festinger L (1950) Informal social communication. Psychol Rev $57: 271-282$ 